\title{
Health Preference Measures in Patients with Obstructive Sleep Apnea Syndrome Undergoing Continuous Positive Airway Pressure Therapy: Data from a Randomized Trial
}

\author{
Fabienne L. Huber ${ }^{a}$ Michael Furian ${ }^{a}$ Malcolm Kohler ${ }^{a}$ Tsogyal D. Latshang ${ }^{b}$ \\ Yvonne Nussbaumer-Ochsner ${ }^{c}$ Alexander Turk ${ }^{d}$ Otto D. Schoch ${ }^{e}$ \\ Irene Laube $^{f}$ Robert Thurnheer ${ }^{g}$ Konrad E. Bloch ${ }^{a}$ \\ aDepartment of Respiratory Medicine, Sleep Disorders Center, University Hospital Zurich, Zurich, Switzerland; \\ bDepartment of Respiratory Medicine, Sleep Disorders Center, Cantonal Hospital Chur, Chur, Switzerland; \\ 'Department of Internal Medicine, Cantonal Hospital Schaffhausen, Schaffhausen, Switzerland; ${ }^{\text {dDepartment of }}$ \\ Internal Medicine, See-Spital, Horgen, Switzerland; 'Department of Respiratory Medicine, Center for Sleep Medicine, \\ Cantonal Hospital St. Gallen, St. Gallen, Switzerland; fDepartment of Respiratory Medicine, Sleep Disorders Center, \\ Triemli Hospital, Zurich, Switzerland; ' ${ }^{9}$ Department of Respiratory Medicine, Sleep Disorders Center, Cantonal \\ Hospital Münsterlingen, Münsterlingen, Switzerland
}

\section{Keywords}

Cost-utility analysis - Health-related quality of life .

Noninvasive ventilation · Sleep apnea

\begin{abstract}
Background: In patients with obstructive sleep apnea syndrome (OSAS), the preference-based, health-related quality of life in terms of utility has not been extensively studied. Objective: To address this point, we compared the performance of different instruments assessing utility in patients with OSAS undergoing continuous positive airway pressure (CPAP) therapy. Materials and Methods: Data of 208 patients with OSAS ( 28 women, mean \pm SE age $54.4 \pm 0.7$ years, apnea-hypopnea index (AHI) $51.9 \pm 1.8 / \mathrm{h}$, Epworth sleepiness score $13.4 \pm 0.2$ ) participating in a randomized trial of different CPAP modalities over 2 years were analyzed. Evaluations included sleep studies, Epworth sleepiness scale, and several utility instruments that measure subjective health preference on a scale ranging from 1 (most preferred and
\end{abstract}

karger@karger.com www.karger.com/res

Karger $\stackrel{\text { ' }}{5}$

GOPEN ACCESS
(C) 2021 The Author(s)

Published by S. Karger AG, Basel

This is an Open Access article licensed under the Creative Commons Attribution-NonCommercial-4.0 International License (CC BY-NC) (http://www.karger.com/Services/OpenAccessLicense), applicable to the online version of the article only. Usage and distribution for commercial purposes requires written permission. perfect health) to 0 (least preferred and very poor health). Results: After 2 years of CPAP therapy, the mean \pm SE AHI was $6.7 \pm 1.5 / \mathrm{h}$ and Epworth score $7.9 \pm 0.4$, both $p<0.001$ versus baseline. Baseline utilities and changes $(95 \%$ confidence interval) after 2 years of CPAP therapy were EuroQol 5-dimensions $0.79 \pm 0.01,0.02(0.00-0.05, p=0.064)$; shortform 6-dimension medical outcome questionnaire $0.72 \pm$ $0.01,0.06(0.04-0.08, p<0.001)$; Euro-thermometer visual analog scale $0.70 \pm 0.01,0.09(0.07-0.12, p<0.001)$; time trade-off $0.82 \pm 0.01,0.03(0.01-0.06, p=0.002)$; and standard gamble $0.82 \pm 0.01,-0.01(-0.03$ to $0.02, p=0.712$ ). Conclusion: The short-form 6-dimensions questionnaire, the Euro-thermometer, and the time trade-off instruments reflected the major clinical improvements in OSAS, while the EuroQoL 5-dimensions and standard gamble tests were not sensitive to CPAP effects. These results indicate that the evaluation of utility of a treatment for OSAS depends critically on the instrument used, which is important from an individual and societal perspective.

(c) 2021 The Author(s)

Published by S. Karger AG, Basel 


\section{Introduction}

Ideally, robust objective outcome measures such as mortality along with patient-centred outcomes such as quality of life measures are used to assess the effectiveness of new treatments [1-4]. Measuring subjective health preferences has become increasingly important in costeffectiveness evaluations because the preference of a population regarding health states serves as a rational basis for decisions on allocation of health-care resources [5]. Various instruments that evaluate subjective health preference, that is, the utility, on a scale from 1 (most preferred and perfect health) to 0 (least preferred and very poor health) have been described [6-8]. By multiplying the utility index with the length of time spent in a health state, so-called quality-adjusted life years (QALYs) can be computed $[1,3,9]$. Based on QALY's and the costs, the incremental cost-effectiveness ratio, an index of the extra costs per QALY gained with a certain therapy, can be determined. The incremental cost-effectiveness ratio is used to support decisions on resource allocation in health-care politics. Since the utility index is an important factor in such calculations, its reliability is crucial. There are different instruments to assess utility either in a direct or indirect way. Direct utility instruments are the standard gamble test [10], the time trade-off test [11], and the Eurothermometer visual analog scale $[12,13]$. Indirect utility indices can be derived from quality of life questionnaires such as the Short Form of the Medical Outcome Questionnaire (SF-36) [14-16] or the EuroQol questionnaire [13], that is, the short-form 6-dimension (SF-6D) and EuroQol 5-dimension (EQ-5D) utility index. As utility is influenced by various factors such as age, sex, cultural, or socioeconomic conditions, they have to be validated for every specific population $[17,18]$.

The obstructive sleep apnea syndrome (OSAS) is a highly prevalent disorder characterized by intermittent collapse of the upper airway during sleep leading to cessation of airflow with cyclic oxygen desaturations and fragmented sleep. Affected patients suffer from excessive daytime sleepiness and cardiovascular disease [19] and are prone to cause sleepiness-related accidents [20]. Apart from life-style modifications [21], different treatments of OSAS are available, among them nocturnal application of continuous positive airway pressure (CPAP) via a mask [22], mandibular advancement devices [23], and upper airway surgery in selected cases. Given the high prevalence of OSAS and its major impact on quality of life, morbidity, and mortality, evaluation of treatments in terms of patient-preference and cost-effectiveness are es- sential. However, the performance of different utility instruments in the evaluation of treatment effectiveness in patients with OSAS over time has not been extensively evaluated [24]. Therefore, the current study compares the performance of various utility instruments in reflecting treatment effects in patients with OSAS participating in a randomized trial of CPAP using 2 pressure setting modes, that is, autoadjusted (autoCPAP) and fixed mask pressure (fCPAP) over the course of 2 years [25]. We evaluated the hypothesis that changes in utility induced by CPAP therapy depended on the instrument used.

\section{Materials and Methods}

This study was part of a Swiss multicenter, randomized, parallelgroup equivalence trial evaluating autoCPAP versus fCPAP in treatment of OSAS over a 2-year period [25]. The co-primary outcomes were sleepiness evaluated by the Epworth sleepiness score (ESS) and the Oxford Sleep Resistance (OSLER) test. Baseline characteristics, co-primary outcomes, apnea-hypopnea index (AHI), oxygen desaturation index (ODI), and SF-6D utility have been reported [25]. The analysis of various utility indices, the focus of the current study, has not been published. Participants gave written informed consent and the protocol was approved by the local Ethics Committees and registered at ClinicalTrials.gov (NCT00280800). This study was supported by the Swiss National Science Foundation, Lunge Zürich, Lungenliga St. Gallen. Sponsors had no role in study design or conduct, data analysis, and manuscript preparation.

\section{Participants}

Men and women, aged 18-75 years, diagnosed with OSAS by a consistent history of excessive daytime sleepiness (ESS $\geq 8$ ) and an AHI $\geq 10 / h$ recorded during a sleep study were invited to undergo CPAP treatment.

\section{Interventions}

After instruction and mask-fitting, participants used autoCPAP with pressure 5-15 mbar for 2-4 weeks. If the average use during the adaptation period was $\geq 2 \mathrm{~h} /$ night and participants wished to continue, they were randomized to either autoCPAP or fCPAP (pressure set at 90th percentile applied by autoCPAP during the adaptation period) and either to a Philips Respironics RemStar or a ResMed AutoSet device. Follow-up was at 1, 3, 12, and 24 months.

\section{Assessments}

Assessments included a medical history, clinical examination, direct and indirect utility instruments described below, ESS [26], Functional Outcome of Sleep Questionnaire (FOSQ) [27], SF-36 [14], and a sleep study.

\section{Utility Instruments [28]}

Of 5 different utility instruments employed, 3 were direct (time trade-off, standard gamble, Euro-thermometer VAS) and 2 indirect (SF-6D and EQ-5D). Patients were instructed in detail before performing tests. 
The Time Trade-Off test measures the theoretical disposition of someone to trade-off years of life for obtaining full health. Using an interactive computer program, participants indicated their preference of either living in the current health state for 20 years (selected time horizon) or to live for a shorter time but in perfect health. The time in perfect health was repeatedly varied until a point of indifference was reached. The utility index was computed as (20 years - number of years traded off)/20 years $[7,11]$.

The Standard Gamble test measures the theoretical willingness of a person to take a certain risk of immediate death for the chance of obtaining lifelong, perfect health. Patients were repeatedly asked to choose between 2 theoretical alternatives: either continue life in the current health state or receive a therapy that provides perfect health but is associated with a certain risk of immediate death. Using a computer application, the risk of immediate death was repeatedly changed until a point of indifference was reached. The probability of perfect health at this point $(=1$-risk of immediate death) was taken as utility index $[7,10]$.

The Euro-Thermometer visual analog scale (VAS) consists of a line on a sheet of paper, $100 \mathrm{~mm}$ in length, and labeled with "very poor health state or death" at one end and "perfect health" at the other end. Participants had to make a mark on the line with a pencil at a position representing their perceived health state. The utility index was obtained by measuring the distance of the mark from the end labeled "very poor health state or death" in $\mathrm{mm}$ and dividing it by $100 \mathrm{~mm}[12,13]$.

The EQ-5D utility index was derived from the EuroQol questionnaire comprising the 5-dimension mobility, self-care, usual activities, pain/discomfort, and anxiety/depression. To derive the EQ-5D utility index, scores from a standard set of preferenceweights from general population samples are applied $[12,13]$.

The SF-6D utility index was obtained from responses to the short-form of the medical outcome questionnaire (the SF-36) [14, 16]. It includes the dimension physical functioning, role limitations, social functioning, bodily pain, mental health, and vitality. SF-6D utility scores were derived by using valuations obtained in a British population.

\section{Main Outcomes and Sample Size}

Main outcomes were the effect sizes of different utility indices. The sample size was computed for the primary outcomes of the main trial, the ESS, and the sleep resistance time [25]. At least 188 participants were required to achieve $80 \%$ power $(\mathrm{alpha}=0.05)$ to detect equivalence within predefined limits of \pm 2 points in ESS and \pm 3 min in sleep resistance time.

\section{Randomization and Masking}

A 1:1 balanced-block randomization was applied. Participants and assessors were blinded to the treatment mode and brand.

\section{Data Analysis and Statistics}

Effects of CPAP treatment were evaluated by an intention-totreat analysis with missing data replaced by multiple imputations $(n=20)$ using regression models with chained equations [29]. Means (SD) of outcomes at baseline and changes versus baseline with $95 \%$ confidence intervals (95\% CI) were computed using multilevel mixed-effects linear regression models with time as predictor. Adjusted analyses were performed with baseline values of the dependent variable, baseline AHI, and ESS. In further, exploratory analyses, the severity of sleep apnea, categorized by terciles
Table 1. Patient characteristics
Men and women, $n(\%)$

Age, years

BMI, $\mathrm{kg} / \mathrm{m}^{2}$

AHI, events/h

Oxygen desaturation index, events $>3 \% / \mathrm{h}$

Nocturnal mean oxygen saturation by pulse oximetry, \%

Score on Epworth sleepiness scale

Means \pm SD. AHI, apnea-hypopnea index
$180(87) ; 28(13)$

$54.4 \pm 10.4$

$33.5 \pm 6.1$

$51.9 \pm 25.6$

$46.4 \pm 24.9$

$91.7 \pm 3.8$

$13.4 \pm 3.5$ of the AHI, the hours of CPAP use, and the CPAP mode were included as predictors in regression models of utility indices. A perprotocol analysis with all available data was also performed. Effect sizes were computed as mean change in any variable divided by the pooled SD with effect sizes $\geq 0.2-0.5$ considered as small, $\geq 0.5-0.8$ as moderate, and $\geq 0.8$ as large [30]. A probability of $p<0.05$ was assumed as statistically significant.

\section{Results}

Of 952 patients assessed, 208 qualified for the study and were randomized. They were predominantly middleaged, mildly obese men with moderate to severe OSAS (Table 1). 36 participants dropped out because of several reasons (shown in Fig. 1). 172 patients, 148 men and 24 women, completed the trial and represented the per-protocol population.

The main outcomes over the course of 2 years are summarized in Table 2 and shown in Figure 2. In both auto$\mathrm{CPAP}$ and fCPAP groups, there were major reductions in ESS and AHI from elevated baseline values (means \pm SD for both groups ESS $13.4 \pm 4.3$, AHI $51.9 \pm 15.9$ events/h) into the normal range within 3 months of CPAP treatment (mean for both groups ESS 7.9 \pm 5.0 , AHI $6.7 \pm 21.6$ events/h, $p<0.001$, both instances). These improvements were associated with a major increase in the SF-36 vitality score (mean \pm SD for both groups at baseline $43.5 \pm$ 27.4 , at 3 months $63.6 \pm 30.3, p<0.001$ ). The favorable effects of CPAP therapy were maintained over the following 18 months until the end of the observation period (shown in Fig. 2). As the treatment mode (autoCPAP and fCPAP) was not a significant predictor of utility indices in regression analyses (see online suppl. Table 1; see www. karger.com/doi/10.1159/000513306 for all online suppl. material), treatment effects are summarized for both groups together in Table 2. Mean utility indices were 


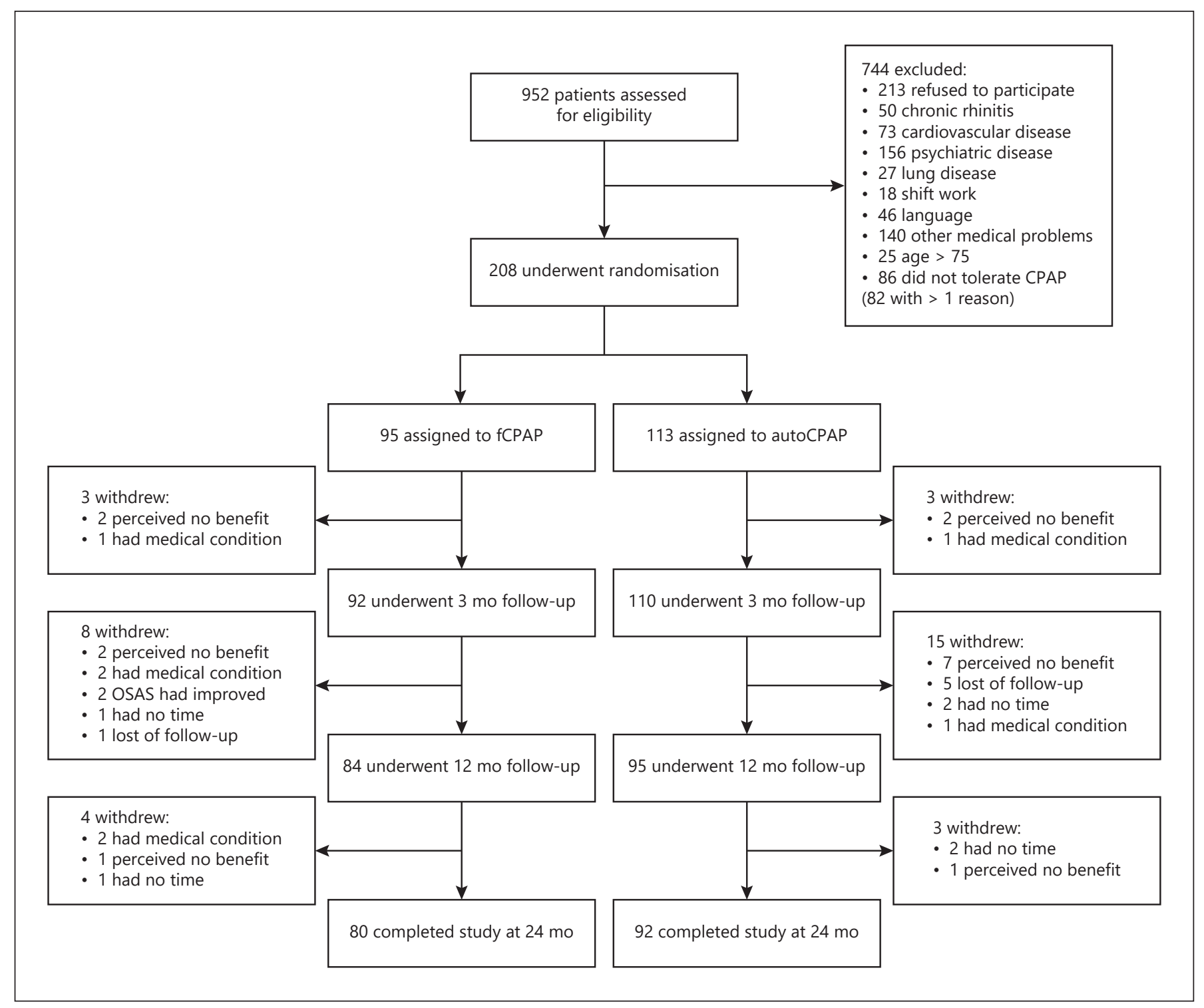

Fig. 1. Participant flow.

0.70-0.82 at baseline. Changes over the course of the subsequent CPAP treatment period were largest for Eurothermometer (increase by $0.08-0.09$ ), SF-6D (increase by $0.05-0.06$ ), and time trade-off utility indices (increase by $0.03-0.04)$. Changes in EQ-5D (-0.01) and standard gamble utility indices (0.02) were not statistically significant. Similar trends as in unadjusted and intention-totreat analyses were seen in adjusted and per-protocol analyses (Tables 2, 3). Effects sizes of CPAP therapy were large to very large $(>0.8)$ for the AHI, ESS, and SF-36 vitality (shown in Fig. 3). Effect sizes were small to moderate $(>0.35)$ for utility indices from Euro-thermometer
VAS, time trade-off, and SF-6D instruments. Effect sizes of utility indices from EQ-5D and standard gamble tests were very small with $95 \%$ CI overlapping zero in most instances (shown in Fig. 3). The lower limits of 95\% CI of effect sizes of utility indices from Euro-thermometer VAS, time trade-off, and SF-6D do not overlap with rather low mean effect sizes of EQ-5D and standard gamble utility indices, suggesting significant differences.

The CPAP use at 3,12 , and 24 months was $5.3 \pm 1.6$, $5.4 \pm 1.7$, and $5.6 \pm 1.8 \mathrm{~h} /$ night, respectively. Exploratory regression analyses did not reveal a significant effect of the hours of CPAP use on any of the utility indices (online 
Table 2. Effect of CPAP therapy - intention-to-treat analysis

\begin{tabular}{|c|c|c|c|c|c|c|c|}
\hline Characteristic & Baseline & $\begin{array}{l}\text { Mean change } \\
(95 \% \mathrm{CI}) \\
3 \text { months-BL }\end{array}$ & $p$ value & $\begin{array}{l}\text { Mean change } \\
(95 \% \mathrm{CI}) \\
12 \text { months-BL }\end{array}$ & $p$ value & $\begin{array}{l}\text { Mean change } \\
\text { ( } 95 \% \mathrm{CI}) \\
24 \text { months-BL }\end{array}$ & $p$ value \\
\hline AHI events/h & $51.9 \pm 15.9$ & $-45.0(-48.1 ;-41.9)$ & $<0.001$ & $-44.1(-47.6 ;-40.7)$ & $<0.001$ & $-43.7(-46.9 ;-40.4)$ & $<0.001$ \\
\hline ODI $>3 \%$ - events $/ \mathrm{h}$ & $46.4 \pm 15.9$ & $-38.2(-41.1 ;-35.3)$ & $<0.001$ & $-37.5(-40.6 ;-34.5)$ & $<0.001$ & $-36.8(-39.8 ;-33.9)$ & $<0.001$ \\
\hline Score on Epworth sleepiness scale & $13.4 \pm 4.3$ & $-5.5(-6.1 ;-4.9)$ & $<0.001$ & $-5.6(-6.2 ;-5.0)$ & $<0.001$ & $-5.7(-6.3 ;-5.1)$ & $<0.001$ \\
\hline SF-36 vitality score & $43 \pm 20.2$ & $20(17 ; 23)$ & $<0.001$ & $20(17 ; 23)$ & $<0.001$ & $19(16 ; 22)$ & $<0.001$ \\
\hline EQ-5D utility index & $0.79 \pm 0.16$ & $0.01(-0.01 ; 0.03)$ & 0.437 & $0.01(-0.01 ; 0.03)$ & 0.436 & $0.02(0.00 ; 0.05)$ & 0.064 \\
\hline EQ-5D utility index, adjusted ${ }^{a}$ & & $0.01(-0.01 ; 0.03)$ & 0.529 & $0.01(-0.01 ; 0.03)$ & 0.547 & $0.02(0.00 ; 0.04)$ & 0.064 \\
\hline SF-6D utility index & $0.72 \pm 0.13$ & $0.05(0.03 ; 0.07)$ & $<0.001$ & $0.05(0.04 ; 0.07)$ & $<0.001$ & $0.06(0.04 ; 0.08)$ & $<0.001$ \\
\hline SF-6D utility index, adjusted ${ }^{a}$ & & $0.05(0.03 ; 0.06)$ & $<0.001$ & $0.05(0.04 ; 0.07)$ & $<0.001$ & $0.06(0.04 ; 0.07)$ & $<0.001$ \\
\hline Euro-thermometer VAS utility index & $0.70 \pm 0.16$ & $0.09(0.06 ; 0.11)$ & $<0.001$ & $0.09(0.06 ; 0.11)$ & $<0.001$ & $0.09(0.07 ; 0.12)$ & $<0.001$ \\
\hline Euro-thermometer VAS utility index, adjusted ${ }^{\mathrm{a}}$ & & $0.08(0.06 ; 0.11)$ & $<0.001$ & $0.09(0.07 ; 0.11)$ & $<0.001$ & $0.09(0.07 ; 0.12)$ & $<0.001$ \\
\hline SG utility index & $0.82 \pm 0.18$ & $0.01(-0.01 ; 0.04)$ & 0.280 & $0.02(-0.01 ; 0.05)$ & 0.119 & $-0.01(-0.03 ; 0.02)$ & 0.712 \\
\hline SG utility index, adjusted ${ }^{\mathrm{a}}$ & & $0.01(-0.01 ; 0.04)$ & 0.187 & $0.02(0.00 ; 0.05)$ & 0.034 & $-0.01(-0.03 ; 0.02)$ & 0.638 \\
\hline TTO utility index & $0.82 \pm 0.17$ & $0.04(0.02 ; 0.06)$ & $<0.001$ & $0.04(0.02 ; 0.06)$ & $<0.001$ & $0.03(0.01 ; 0.06)$ & 0.002 \\
\hline TTO utility index, adjusted ${ }^{\mathrm{a}}$ & & $0.04(0.02 ; 0.06)$ & $<0.001$ & $0.04(0.02 ; 0.07)$ & $<0.001$ & $0.04(0.02 ; 0.06)$ & $<0.001$ \\
\hline
\end{tabular}

ODI, oxygen desaturation index $>3 \%$ dips; SF-36, short-form medical outcome questionnaire; EQ-5D, EuroQol 5-dimensions questionnaire; SF-6D, short-form 6 dimension; VAS, visual analog scale; BL, baseline; CPAP, continuous positive airway pressure; AHI, apnea-hypopnea index; TTO, time tradeoff. a Adjusted for baseline values of the corresponding variable.

Table 3. Effect of CPAP therapy - per-protocol analysis

\begin{tabular}{|c|c|c|c|c|c|c|c|}
\hline Characteristic & Baseline & $\begin{array}{l}\text { Mean change } \\
(95 \% \mathrm{CI}) \\
3 \text { months-BL }\end{array}$ & $p$ value & $\begin{array}{l}\text { Mean change } \\
(95 \% \mathrm{CI}) \\
12 \text { months-BL }\end{array}$ & $p$ value & $\begin{array}{l}\text { Mean change } \\
(95 \% \mathrm{CI}) \\
24 \text { months-BL }\end{array}$ & $p$ value \\
\hline ODI $>3 \%-$ events $/ \mathrm{h}$ & $46.4 \pm 14.4$ & $-38.4(-41.0 ;-35.9)$ & $<0.001$ & $-39.4(-42.0 ;-36.8)$ & $<0.001$ & $-39.6(-42.3 ;-36.9)$ & $<0.001$ \\
\hline Score on Epworth sleepiness scale & $13.4 \pm 3.6$ & $-5.5(-6.0 ;-5.0)$ & $<0.001$ & $-5.9(-6.4 ;-5.4)$ & $<0.001$ & $-6.2(-6.7 ;-5.7)$ & $<0.001$ \\
\hline SF-36 vitality score & $43 \pm 14$ & $20(18 ; 23)$ & $<0.001$ & $21(19 ; 24)$ & $<0.001$ & $19(17 ; 22)$ & $<0.001$ \\
\hline SF-6D utility index & $0.72 \pm 0.13$ & $0.05(0.03 ; 0.06)$ & $<0.001$ & $0.05(0.04 ; 0.07)$ & $<0.001$ & $0.06(0.04 ; 0.07)$ & $<0.001$ \\
\hline SF-6D utility index, adjusted ${ }^{\mathrm{a}}$ & & $0.05(0.03 ; 0.06)$ & $<0.001$ & $0.05(0.04 ; 0.07)$ & $<0.001$ & $0.06(0.04 ; 0.07)$ & $<0.001$ \\
\hline Euro-thermometer VAS utility index & $0.70 \pm 0.16$ & $0.08(0.06 ; 0.11)$ & $<0.001$ & $0.09(0.07 ; 0.11)$ & $<0.001$ & $0.09(0.07 ; 0.11)$ & $<0.001$ \\
\hline Euro-thermometer VAS utility index, adjusted & & $0.08(0.06 ; 0.11)$ & $<0.001$ & $0.09(0.07 ; 0.11)$ & $<0.001$ & $0.09(0.07 ; 0.12)$ & $<0.001$ \\
\hline SG utility index & $0.82 \pm 0.18$ & $0.01(-0.01 ; 0.03)$ & 0.273 & $0.02(0.00 ; 0.04)$ & 0.080 & $-0.01(-0.03 ; 0.01)$ & 0.407 \\
\hline
\end{tabular}

ODI, oxygen desaturation index $>3 \%$ dips; SF-36, short-form medical outcome questionnaire; EQ-5D, EuroQol 5-dimensions questionnaire; SF-6D, short-form 6 dimension; VAS, visual analog scale; BL, baseline; CPAP, continuous positive airway pressure; AHI, apnea-hypopnea index; TTO, time tradeoff; SG, standard gamble. ${ }^{a}$ Adjusted for baseline values of the corresponding variable.

suppl. Table 2). To evaluate potential effects of sleep apnea severity on utility indices, patients were divided into 3 groups according to $\mathrm{AHI}$ terciles $(\mathrm{AHI}>10-37 / \mathrm{h}=$ 1st tercile, AHI $>37-62.8 / \mathrm{h}=2$ nd tercile, and AHI 63$136 / \mathrm{h}=3 \mathrm{rd}$ tercile). In regression analysis, AHI terciles were not significantly associated with utility indices over the course of the CPAP treatment period with exception of slightly higher standard gamble utility indices at 24 months in patients with more severe (2nd and 3rd AHI terciles) compared to milder sleep apnea (1st AHI tercile) (online suppl. Table 3). 

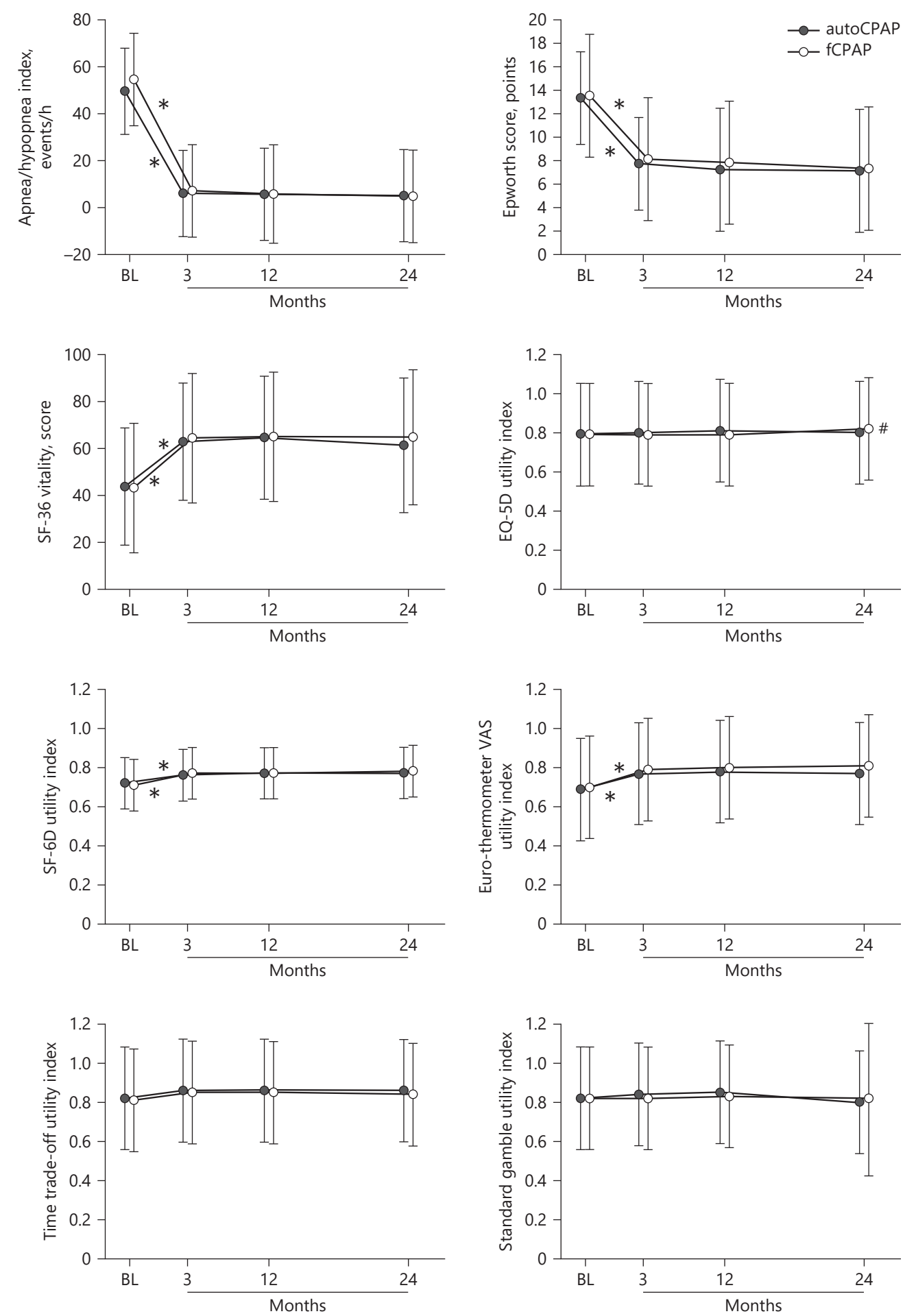

Fig. 2. Effects of CPAP therapy on mean (SD) main outcomes in patients with the over the course of 2 years. Results are not statistically different for patients randomized to autoCPAP and fCPAP, and main effects were achieved within the first 3 months of treatment. ${ }^{*} p<0.05$ for changes of autoCPAP and fCPAP at 3 months versus baseline and $\# p<0.05$ for changes of fCPAP at 24 versus 3 months. CPAP, continuous positive airway pressure; fCPAP, fixed CPAP; auotCPAP, autoadjusted CPAP; OSAS, obstructive sleep apnea syndrome. 
Fig. 3. Plot of mean effect sizes with $95 \%$ CIs of CPAP therapy compared to pretreatment baseline. Each panel shows results after 3, 12, and 24 months of CPAP therapy. AHI, apnea-hypopnea index; ESS, Epworth sleepiness scale; SF-36_vt, shortform medical outcome questionnaire vitality score; EQ-5D, EuroQol 5-dimension utility index; SF-6D, short-form 6-dimension utility index; VAS, Euro-thermometer visual analog scale utility index; TTO, time trade-off utility index; SG, standard gamble utility index; CPAP, continuous positive airway pressure; AHI, apnea-hypopnea index; CI, confidence interval.

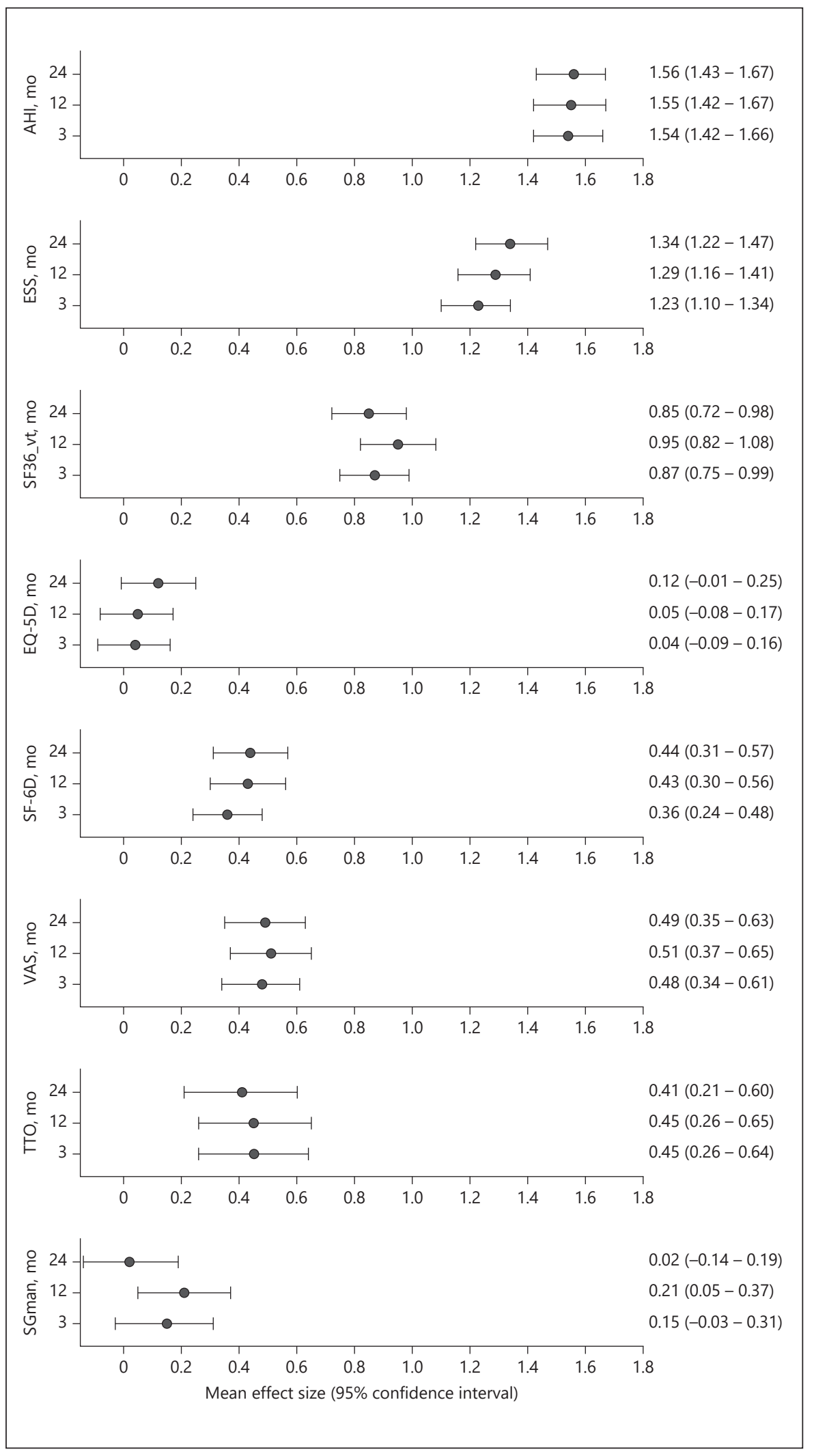




\section{Discussion}

The current study is the first to systematically investigate the response of several utility instruments to CPAP therapy over the course of 2 years in a large group of patients with OSAS participating in a randomized trial of different CPAP modalities. We found that only 3 of 5 commonly used utility indices, that is, those derived by the Euro-thermometer VAS, the SF-6D, and the time trade-off test, were responsive to CPAP therapy with small to moderate effect sizes. In contrast, 2 other commonly used utility indices, the EQ-5D and the standard gamble test, did not significantly change with CPAP therapy even though patients revealed major improvements in symptoms and in AHI which are important metrics of OSAS severity and response to treatment. Our results suggest that it is essential to account for the instrument used to evaluate quality of life in terms of utility when assessing effects of disease and its treatment in patients with OSAS.

An analysis of the literature reveals that utility instruments have not been extensively evaluated in patients with OSAS. Study designs, utility instruments used, and results were quite heterogeneous.

In one study, 19 patients with OSAS (mean baseline AHI of 67/h) retrospectively rated utility before initiation of therapy as 0.63 and as 0.87 after 9.5 months on CPAP [31]. In another study in 32 patients with OSAS (mean baseline AHI of $55 / \mathrm{h}$ ), the standard gamble utility was much lower, that is, 0.32 , and improved by 0.23 after 3 months of CPAP therapy [32]. In the current trial in 208 patients with a mean baseline AHI of $51.9 / \mathrm{h}$, the pretreatment standard gamble utility was higher $(0.82$, Table 2$)$ than that in the cited studies [31,32] and did not change with treatment even though sleepiness and AHI improved significantly (very large effect sizes of 1.33 in ESS and of 1.56 in AHI, shown in Fig. 3) confirming effective treatment. When applying the time trade-off test to the same patients, baseline utility was also 0.82 , and CPAP therapy improved utility by 0.03 at 24 months corresponding to a small effect size. In a previous study in 66 untreated OSAS patients, we recorded even higher utility indices using the standard gamble (0.97) and time tradeoff (0.94) tests [28]. To our knowledge, no other studies have evaluated the effect of CPAP therapy in OSAS patients using the classical standard gamble or time tradeoff utility tests. In our experience, both tests require extensive explanations to patients and represent a major challenge to their capability of imagination. Therefore, and based on the limited available data showing a large

Health Preference Measures in Sleep Apnea variability in untreated patients and no consistent treatment effect, these tests seem not well suited for application in OSAS patients.

The EQ-5D questionnaire, an indirect utility instrument recommended for valuing health effects by the British NICE guidelines [3], has been employed in OSAS patients with variable results. In a Spanish survey in 46 patients (baseline AHI 41.3/h), the EQ-5D utility index was 0.74 and increased after 3 months of CPAP therapy by 0.07 [33]. In a study in 71 British OSAS patients, a minor improvement in EQ-5D utility of 0.04 was observed after 3 months of CPAP treatment, while standard gamble utility improvement was larger (0.23) [32]. In contrast to these positive effects of CPAP on EQ-5D utility, 4 studies including $>560$ British patients with OSAS [34] did not observe significant improvements in EQ-5D utility with CPAP (472 patients) [34-36] or a mandibular advancement appliance (90 patients) [37]. Since one large study included 278 patients older than 65 years [34], age may have modified the outcome. In 2 of the cited studies [34, 37], the SF-6D utility instrument was also employed and revealed a positive treatment effect. In the current study, no significant effect of CPAP on EQ-5D utility was found while SF-6D utility improved by 0.06 over the course of 2 years. In 95 Brazilian OSAS patients, an increase in SF-6D utility of 0.09 after 1 year of CPAP therapy has been reported [38]. Thus, of the 2 indirect utility instruments (EQ-5D and SF-6D), the EQ$5 \mathrm{D}$ did not consistently capture the improvement in well-being achieved in OSAS patients with CPAP therapy. Presumably, the dimensions of quality of life addressed by the EQ-5D (i.e., mobility, self-care, usual activities, pain/discomfort, and anxiety/depression) are not changing significantly with the treatment of OSAS and/or are not affected by the disorder to a major degree [13]. In turn, the SF-6D seems to include aspects of quality of life such as mental health and vitality that are quite relevant for patients with OSAS and that have shown major treatment effects in various randomized trials evaluating efficacy of CPAP therapy [15].

The Euro-thermometer VAS has been incorporated into the EuroQoL questionnaire as a more direct utility instrument than the EQ-5D. Similar to the direct utility instruments (standard gamble and time trade-off) the Euro-thermometer VAS does not evaluate specific quality of dimensions but is not preference-based. In one study in OSAS patients [32], 3 months of CPAP therapy improved the Euro-thermometer VAS by 0.11 , the standard gamble utility by 0.23 , and the EQ-5D by 0.04 . In the current study, a similar improvement in the Euro-thermometer 
VAS of 0.09 was observed but no significant change in the EQ-5D and the standard gamble utilities (Table 2).

In a previous study, patients seem not to have considered OSAS as a directly life-threatening condition and, therefore, were not willing to trade-off years of life as reflected in high utility indices in time trade-off and standard gamble tests of 0.8-0.9 [28]. In contrast, OSAS patients in another study rated standard gamble utility much lower (0.32), similar to patients suffering from a stroke. Nevertheless, the EQ-5D utility index in the same OSAS patients was considerably higher (0.73) [32]. In the current study, the mean EQ-5D utility index in OSAS patients was even higher (0.79) exceeding also corresponding values reported previously in patients with chronic obstructive pulmonary disease (COPD, 0.644) [39] and patients with various chronic diseases [40] such as osteoporosis (0.59), COPD (0.62), leg ulcer (0.62), lower back pain (0.69), and irritable bowel syndrome (0.73). Of note, in the cited study [40], EQ-5D utility indices were lower (by a mean of 0.05) than SF-6D utility indices, while the current study revealed lower SF-6D than EQ-5D utility indices (mean difference 0.07). Available evidence, therefore, suggests that subjective health preference may be modified by the instrument used for assessment, as well as by age, social and cultural conditions, challenging the concept of utility as a universal, and generic measure allowing uncritical comparison of health state preferences among patients with different diseases.

The perception of health state and performance of utility instruments may also be affected by impairments resulting from OSAS itself [17]. Thus, excessively sleepy OSAS patients may not care or may not be vigilant enough to reliably complete utility tests of quite different complexity such as the standard gamble test and the Eurothermometer VAS. These drawbacks may change with improvement of OSAS with CPAP therapy. However, we did not observe a significant effect of sleep apnea severity over the course of the CPAP treatment period on any of the utility indices with exception of slightly higher time trade-off utility indices at 24 months in patients with severe compared to patients with milder sleep apnea (online suppl. Table 3). Further analyses did not reveal an association of the nightly CPAP use with utility indices (online suppl. Table 2), possibly related to the high treatment adherence in the current study. To our knowledge, the minimally important difference in utility indices has not been conclusively determined. Thus, in studies involving a total of 940 patients with COPD [41], the minimally important difference was found to be 0.051 for EQ5D and 0.069 for VAS utility. Based on data from 8 stud- ies in 323 patients with various diseases [42] (leg ulcer, back pain, limb reconstruction, irritable bowel syndrome, myocardial infarction, osteoarthritis, rheumatoid arthritis, and COPD), the authors suggested a minimally important difference in EQ-5D utility of 0.074 and SF-6D utility of 0.041 ; corresponding effect sizes were small to moderate (0.30 and 0.43). In the current study, effect sizes of CPAP on utility in patients with OSAS were of similar magnitude (0.41-0.49) when evaluated by VAS, SF$6 \mathrm{D}$, and time trade-off instruments. In analogy to the cited studies, we, therefore, propose that these instruments were suitable to capture a minimally important effect of CPAP on health state preference in patients with OSAS, while the EQ-5D and standard gamble instruments were not significantly responsive to the treatment.

Inclusion criteria for the current study selected a phenotype of patients with moderate to severe OSAS without major comorbidities such as cardiovascular, respiratory, or psychiatric disease which might limit the generalizability of the results. It is uncertain whether such comorbidities may affect subjective health preference in patients with OSAS. The current study did not include a randomized control group receiving a placebo intervention or no CPAP therapy. While this may have affected the quantitative assessment of CPAP effects on utility and other outcomes, the comparison among different utility instruments, the main goal of the study, was still valid. The repeated assessments over the course of 2 years have created a unique dataset allowing to compare the responsiveness of different utility instruments to CPAP therapy in OSAS patients over a much longer period than in previous studies. This strength of our study may have entailed the potential drawback of influencing the motivation of patients and test performance.

In conclusion, the current study provides a unique comparison among the performance of 5 different utility instruments evaluated in a large group of patients with OSAS over the first 2 years of CPAP therapy. Only 3 of the instruments (the SF-6D, the Euro-thermometer VAS, and the time trade-off test) were responsive to CPAP treatment, while 2 (including the widely used EQ$5 \mathrm{D}$ and the standard gamble test) remained unchanged despite major improvements in breathing disturbances and symptoms. Since OSAS is a highly prevalent disease associated with significant morbidity and mortality, our findings represent important novel information essential for cost-utility estimations and decisions on treatment of OSAS both from an individual and a societal perspective. 


\section{Statement of Ethics}

The research was conducted ethically in accordance with the World Medical Association Declaration of Helsinki. The study participants have given their written informed consent, and this study protocol was approved by the institute's committee on human research.

\section{Conflict of Interest Statement}

K.E.B. reports grants to his institution from Swiss National Science Foundation, Zurich Lung League, Respironics Foundation, ResMed Switzerland, during the conduct of the study. Dr. Kohler reports personal fees from Bayer, Novartis, GSK, Astra Zeneca, Boehringer Ingelheim, Mundipharma, and outside the submitted work. The other authors report no competing interests.

\section{Funding Sources}

This study was supported by the Swiss National Science Foundation, the Lung Leagues of Zurich, St. Gallen and Thurgau, Switzerland. The Respironics Foundation and ResMed Switzerland have provided unconditional institutional grants for the study. This was an investigator-initiated study; sponsors had no role in design or conduct of the study, data analysis, and preparation of the manuscript.

\section{Author Contributions}

F.H. contributed to data analysis and interpretation and drafted the article. M.F. contributed to data analysis and interpretation. M.K., T.D.L., Y.N.-O., A.T., O.D.S., I.L., and R.T. contributed to acquisition, analysis, and interpretation of data. K.E.B. contributed to conception and design of the study, acquisition, analysis, interpretation of data, and drafting the article. All authors reviewed and commented on the manuscript.

\section{References}

1 Weinstein MC, Stason WB. Foundations of cost-effectiveness analysis for health and medical practices. N Engl J Med. 1977; 296(13):716-21.

2 Stein K, Fry A, Round A, Milne R, Brazier J. What value health?: a review of health state values used in early technology assessments for NICE. Appl Health Econ Health Policy. 2005;4(4):219-28.

3 Guide to the methods of technology appraisal 2013. Available from: https://www.nice.org. $\mathrm{uk} / \mathrm{process} / \mathrm{pmg} 9 /$ resources/guide-to-themethods-of-technology-appraisal2013-pdf-2007975843781; nice.org.uk/process/pmg9.

4 Whitehead SJ, Ali S. Health outcomes in economic evaluation: the QALY and utilities. Br Med Bull. 2010;96:5-21.

5 Tsevat J. What do utilities measure? Med Care. 2000;38(9 Suppl):II160-4.

6 Tengs TO, Wallace A. One thousand healthrelated quality-of-life estimates. Med Care. 2000;38(6):583-637.

7 Garza AG, Wyrwich KW. Health utility measures and the standard gamble. Acad Emerg Med. 2003;10(4):360-3.

8 Riethmuller S, Muller-Wenk R, Knoblauch A, Schoch OD. Monetary value of undisturbed sleep. Noise Health. 2008;10:46-54.

9 Sanders GD, Maciejewski ML, Basu A. Overview of cost-effectiveness analysis. JAMA. 2019;321(14):1400-1.

10 von Neumann J, Morgenstern O. Theory of games and economic behavior. 60th Anniversary ed. Princeton, NJ: Princeton University Press; 2007.
11 Torrance GW, Thomas WH, Sackett DL. A utility maximization model for evaluation of health care programs. Health Serv Res. 1972; $7(2): 118-33$.

12 Torrance GW, Feeny D, Furlong W. Visual analog scales: do they have a role in the measurement of preferences for health states? Med Decis Making. 2001;21(4):329-34.

13 Brooks R, Rabin R, De Cahrro F. The measurement and valuation of health status using the EQ-5D: a European perspective. London: Kluwer Academic Publishers; 2003.

14 Ware JE Jr, Snow KK, Kosinski M, Gandek B. SF-36 health survey. Manual and interpretation guide. Boston: The Medical Outcome Trust, New England Medical Center; 1993.

15 Brazier J, Roberts J, Deverill M. The estimation of a preference-based measure of health from the SF-36. J Health Econ. 2002;21(2): 271-92.

16 Brazier J, Usherwood T, Harper R, Thomas K. Deriving a preference-based single index from the UK SF-36 Health Survey. J Clin Epidemiol. 1998;51(11):1115-28.

17 Dolan P, Gudex C, Kind P, Williams A. The time trade-off method: results from a general population study. Health Econ. 1996;5(2): 141-54.

18 Greiner W, Weijnen T, Nieuwenhuizen M, Oppe S, Badia X, Busschbach J, et al. A single European currency for EQ-5D health states. Results from a six-country study. Eur J Health Econ. 2003;4(3):222-31.

19 Maeder MT, Schoch OD, Rickli H. A clinical approach to obstructive sleep apnea as a risk factor for cardiovascular disease. Vasc Health Risk Manag. 2016;12:85-103.

20 Jordan AS, McSharry DG, Malhotra A. Adult obstructive sleep apnoea. Lancet. 2014; 383(9918):736-47.
21 Randerath WJ, Verbraecken J, Andreas S, Bettega G, Boudewyns A, Hamans E, et al. Non-CPAP therapies in obstructive sleep apnoea. Eur Respir J. 2011;37(5):1000-28.

22 McDaid C, Durée KH, Griffin SC, Weatherly HL, Stradling JR, Davies RJ, et al. A systematic review of continuous positive airway pressure for obstructive sleep apnoea-hypopnoea syndrome. Sleep Med Rev. 2009;13(6): 427-36.

23 Bratton DJ, Gaisl T, Schlatzer C, Kohler M. Comparison of the effects of continuous positive airway pressure and mandibular advancement devices on sleepiness in patients with obstructive sleep apnoea: a network meta-analysis. Lancet Respir Med. 2015;3(11): 869-78.

24 Wickwire EM, Albrecht JS, Towe MM, Abariga SA, Diaz-Abad M, Shipper AG, et al. The impact of treatments for OSA on monetized health economic outcomes: a systematic review. Chest. 2019;155(5):947-61.

25 Bloch KE, Huber F, Furian M, Latshang TD, Lo Cascio CM, Nussbaumer-Ochsner Y, et al. Autoadjusted versus fixed CPAP for obstructive sleep apnoea: a multicentre, randomised equivalence trial. Thorax. 2018;73(2):174-84.

26 Bloch KE, Schoch OD, Zhang JN, Russi EW. German version of the Epworth Sleepiness Scale. Respiration. 1999;66(5):440-7.

27 Weaver TE, Laizner AM, Evans LK, Maislin G, Chugh DK, Lyon K, et al. An instrument to measure functional status outcomes for disorders of excessive sleepiness. Sleep. 1997; 20(10):835-43.

28 Schmidlin M, Fritsch K, Matthews F, Thurnheer R, Senn O, Bloch KE. Utility indices in patients with the obstructive sleep apnea syndrome. Respiration. 2010;79(3):200-8. 
29 White IR, Royston P, Wood AM. Multiple imputation using chained equations: issues and guidance for practice. Stat Med. 2011; 30(4):377-99.

30 Kazis LE, Anderson JJ, Meenan RF. Effect sizes for interpreting changes in health status. Med Care. 1989;27(3 Suppl):S178-89.

31 Tousignant $\mathrm{P}$, Cosio MG, Levy RD, Groome PA. Quality adjusted life years added by treatment of obstructive sleep apnea. Sleep. 1994; 17(1):52-60.

32 Chakravorty I, Cayton RM, Szczepura A. Health utilities in evaluating intervention in the sleep apnoea/hypopnoea syndrome. Eur Respir J. 2002;20(5):1233-8.

33 Mar J, Rueda JR, Durán-Cantolla J, Schechter C, Chilcott J. The cost-effectiveness of nCPAP treatment in patients with moderate-to-severe obstructive sleep apnoea. Eur Respir J. 2003;21(3):515-22.
34 McMillan A, Bratton DJ, Faria R, LaskawiecSzkonter M, Griffin S, Davies RJ, et al. Continuous positive airway pressure in older people with obstructive sleep apnoea syndrome (PREDICT): a 12-month, multicentre, randomised trial. Lancet Respir Med. 2014;2(10): 804-12.

35 Jenkinson C, Stradling J, Petersen S. How should we evaluate health status? A comparison of three methods in patients presenting with obstructive sleep apnoea. Qual Life Res. 1998;7(2):95-100.

36 Jenkinson C, Stradling J, Petersen S. Comparison of three measures of quality of life outcome in the evaluation of continuous positive airways pressure therapy for sleep apnoea. J Sleep Res. 1997;6(3):199-204.

37 Quinnell TG, Bennett M, Jordan J, Clutterbuck-James AL, Davies MG, Smith IE, et al. A crossover randomised controlled trial of oral mandibular advancement devices for obstructive sleep apnoea-hypopnoea (TOMADO). Thorax. 2014;69(10):938-45.
38 Rizzi CF, Ferraz MB, Poyares D, Tufik S. Quality-adjusted life-years gain and health status in patients with OSAS after one year of continuous positive airway pressure use. Sleep. 2014;37(12):1963-8.

39 Chen J, Wong CK, McGhee SM, Pang PK, Yu WC. A comparison between the EQ-5D and the SF-6D in patients with chronic obstructive pulmonary disease (COPD). PLoS One. 2014;9(11):e112389.

40 Brazier J, Roberts J, Tsuchiya A, Busschbach J. A comparison of the EQ-5D and SF-6D across seven patient groups. Health Econ. 2004;13(9):873-84.

41 Nolan CM, Longworth L, Lord J, Canavan JL, Jones SE, Kon SS, et al. The EQ-5D-5L health status questionnaire in COPD: validity, responsiveness and minimum important difference. Thorax. 2016;71(6):493-500.

42 Walters SJ, Brazier JE. Comparison of the minimally important difference for two health state utility measures: EQ-5D and SF6D. Qual Life Res. 2005;14(6):1523-32. 